\title{
Stability Analysis for Yield and Yield Attributing Traits in Rice (Oryza sativa L.)
}

\author{
B. Manjunatha*, C. Malleshappa and B. Niranjana Kumara
}
Department of Genetics and Plant Breeding, Agricultural and Horticultural Research Station, Kathalagere- 577219 (Karnataka), India (University of Agricultural and Horticultural Sciences, Shivamogga-577204, Karnataka, India)
*Corresponding author

\section{A B S T R A C T}

\begin{tabular}{|l|}
\hline Ke y w o r d s \\
Stability, Linear, \\
Genotype $\mathrm{x}$ \\
Environment, \\
Variance
\end{tabular}

\section{Introduction}

Rice (Oryza sativa L.), belongs to the family Graminae, recognized as "Millennium Crop" expected to contribute towards food security in the world, as it is one of the staple cereal crops of the world and a primary source of food for more than half the world's population. With an alarming increase in the population throughout the world, the demand for rice will continue to increase in near future. Therefore, rice breeders across the world aim at increasing the grain yield of rice
(Song et al., 2007). Worldwide, rice is cultivated in an area of about 161.4 million hectares, production of about 506.3 million tonnes and productivity of 3.14 tonnes per hectare. In India area under rice cultivation is 44.11 million hectare and production of about 105.48 million tonnes with 2.39 tonnes per hectare productivity. In Karnataka, it is grown in an area of 13.26 lakh hectares with production of 3541 thousand tons and productivity of 2.67 tonnes per ha (Annon, 2016). Yield is a complex quantitative character and is greatly influenced by 
environmental fluctuations; hence, the selection for superior genotypes based on yield per se at a single location in a year may not be very effective (Shrestha et al., 2012). The assessment of stability of a genotype under different environments is useful for recommending cultivars for known conditions of cultivation. The stability of varieties over wide range of environments with high yield potential is desirable. It has always been emphasized by breeders as base before releasing an ideal variety for commercial cultivation (Singh and Shukla, 2001). For a genotype to be commercially successful, it must perform well across the range of environment likely to be encountered in a target region over the entire array of years in which the genotype could be in use. Beyond seasonal and location differences, however, cultivation conditions within season do transit from one condition to the other, as dictated by variability in moisture and other environmental indices.

The presence of $\mathrm{G} \times \mathrm{E}$ interaction is naturally makes it difficult to fully realise the potential of a genotype for a region in which weather varies from year to year. When the $G \times E$ interaction is significant, the plant and environmental factors that play a major role in causing differential performance, and their significance in determining desirable breeding strategies, must be carefully considered (Kang and Martin, 1987 and Yan and Hunt, 2000). Understanding the genotype $\mathrm{x}$ environment interaction has long been a key issue for plant breeders and geneticists. In crop performance, the observed phenotype is a function of genotype $(\mathrm{G})$, environment $(\mathrm{E})$ and genotype $x$ environment interaction (GEI). GEI is said to occur when different cultivars or genotypes respond differently to diverse environments. Researchers agree that GEI is important only when it is significant and causes considerable changes in genotype ranks in different environments. If this interaction is more, then the stable performance of the variety is less and vice versa. Hence, testing of newly developed genotypes for their stable performance is vital across the different environments.

\section{Materials and Methods}

The experimental material for the contemporary study comprises of twenty advanced breeding lines of $\mathrm{F}_{6}$ generation (Table 1.) with three checks Jyothi, KHP-2 and Tunga collected from Department of Genetics and Plant breeding, College of Agriculture Shivamogga. The research was carried out during kharif 2016. The experiment was laid out in Randomized Complete Block Design (RCBD) with two replications in puddle field at all locations (Table 2). Five plants in all the advanced breeding lines were selected at random from each replication for recording of observations on metric characters of these advanced breeding lines were used for recording all the below cited characters. The average of observations recorded on these five plants was considered for statistical analysis. Similarly plant morphological characters of each genotype were recorded by selecting single or group of plants depending on all characters at different stages of crop growth. Days to fifty per cent flowering, Days to maturity, Plant height $(\mathrm{cm})$, Panicle length $(\mathrm{cm})$, Number of tillers, Number of productive tillers per plant, Number of spikelets per plant, Number of grains per panicle, Panicle fertility (per cent), Test weight (g), Grain yield (kg/ha), Straw yield (kg/ha) and Harvest Index (\%).To analyze the data over six environments the stability model proposed by Eberhart and Russel (1966) have proposed a dynamic approach for studying and interaction phenotypic stability from regression analysis. It enables selection of genotypes that may reasonably show stable performance over a range of environment was adopted. 


\section{Results and Discussion}

In the present study stability parameters such as mean $(\mu)$, regression coefficient (bi) and deviation from regression $\left(\mathrm{S}^{2} \mathrm{di}\right)$, as suggested by Eberhart and Russel (1966) were considered to explain and discuss the stability of different advanced breeding lines for various characters under consideration. From the pooled analysis of variance, it was evident that the significant mean squares due to environment (linear) for grain yield revealed differential response of the genotypes and environment, mean square for pooled deviation were significant for all most all the characters studied (Table 3). The results supported with the reports of Vanave et al., 2014.

Table 4 shows that the advanced breeding lines JB-1-22-2 and JA-4-3 had less mean value for days to fifty per cent flowering than the population mean had regression coefficient unity and least deviation from regression, indicating that with respect to days to fifty per cent flowering, these advanced breeding lines show stable performance across the environments.

So, by using these advanced breeding lines in breeding programme can develop medium duration or short duration cultivars. JK-1-118, JA-4-2, JB-1-22-2, JA-6-3, JA-6-4 and JA6-2 are identified as stable lines for specific locations these results were in associated with Basavaraj (1994) and Subramanya (1996). In other hand Koli et al., (2015) reported that days to fifty per cent flowering is a stable character across the environment. The advanced breeding line JB-1-20-2 had less mean value for days to maturity than population mean, also had regression coefficient value is around unity and less deviation from regression. So, it is indicated that this advanced breeding line had stable performance across the environments and less sensitive to environment it can adapt to the diverse environments. These findings are agreement with those of Sawant et al., (2006) and Praveen et al., (2013). JA-4-2 and JA-6-2, JA-4-3, JA-6-2, JA-4-2, JA-6-2 and JA-4-2 identified as stable lines for specific locations. The advanced breeding line JT-2-16-1 had more mean value for plant height than the population mean also had regression coefficient value is around unity and less deviation from regression. Significant regression co-efficient was observed for JT153 and JK2 15-2 indicate that they were highly sensitive to environmental changes and rest of the advanced breeding lines had average stability. Basavaraj (1994) also identified advanced breeding lines with average responsiveness and also advanced breeding lines with higher environmental sensitivity.

Subramanya (1996) noted unpredictability of the genotypes for this trait. Similar results were also reported by Koli et al., (2015). JT-216-1, JT-2-16-1, JT-2-22-5, JT-2-16-1, JT-222-5 and JT-2-16-1 are identified as stable lines for specific locations. The advanced breeding line JK-1-12-1 had more mean value than the population mean and also had regression coefficient value is around unity and less deviation from regression.

Similar results were reported Mahapatra and Sujathadas (1999). JT-2-16-1, JT-2-16-1, JB1-11-7, JA-6-4, JA-6-2 and JA-6-4 identified as stable lines for specific locations. High mean values than the population mean, regression coefficient around unity and least deviation from regression were recorded for number of tillers per plant in the advanced breeding lines JB-1-11-7 and JA-6-3 indicating that their stability over wide range of environments. These findings are agreement with those of Umadevi et al., (2008). JA-6-2, JK-1-7-5, JT-2-15-1, JT-2-161, JK-1-13-2 and JT-2-16-1 are identified as stable lines for specific locations. 
Table.1 List of advanced breeding lines $\left(\mathrm{F}_{6}\right)$ used under present investigation including checks

\begin{tabular}{|c|c|c|c|c|}
\hline Cross combinations & Code & $\begin{array}{l}\text { Advanced breeding } \\
\text { lines }\end{array}$ & Grain shape & Grain color \\
\hline \multirow{5}{*}{ JYOTI x BILIYA } & G1 & JB-1-11-7 & Medium slender & Light red \\
\hline & G2 & JB-1-20-2 & Medium slender & Light red \\
\hline & G3 & JB-1-22-1 & Medium slender & Light red \\
\hline & G4 & JB-1-22-2 & Medium slender & Light red \\
\hline & G5 & JB-1-22-3 & Medium slender & Light red \\
\hline \multirow{6}{*}{ JYOTI x KESARI } & G6 & JK-1-7-5 & Medium bold & Dark red \\
\hline & G7 & JK-1-11-8 & Medium bold & Light red \\
\hline & G8 & JK-1-12-1 & Medium bold & Light red \\
\hline & G9 & JK-1-13-1 & Medium bold & Light red \\
\hline & G10 & JK2-2-1-8-1 & Medium bold & Light red \\
\hline & G11 & JK2-1-12-1 & Medium bold & Light red \\
\hline \multirow{6}{*}{ JYOTI x AKKALU } & G12 & JA-4-1 & Medium slender & Light red \\
\hline & G13 & JA-4-2 & Medium slender & Light red \\
\hline & G14 & JA-4-3 & Medium slender & Light red \\
\hline & G15 & JA-6-2 & Medium slender & Light red \\
\hline & G16 & JA-6-3 & Medium slender & Light red \\
\hline & G17 & JA-6-4 & Medium slender & Light red \\
\hline \multirow{3}{*}{ JYOTI x TUNGA } & G18 & JT-2-15-1 & Medium slender & Light red \\
\hline & G19 & JT-2-16-1 & Medium slender & Light red \\
\hline & G20 & JT-2-22-5 & Medium slender & white \\
\hline JYOTHI & G21 & & Bold & $\overline{\text { Red }}$ \\
\hline KHP-2 & G22 & & Slender & Red \\
\hline TUNGA & G23 & & Bold & white \\
\hline
\end{tabular}

Table.2 Location of experiments conducted to evaluate rice genotypes for stability analysis

\begin{tabular}{|c|c|c|c|c|c|c|c|}
\hline SL. & Particulars & \multicolumn{6}{|c|}{ Environments } \\
\hline 1 & Locations & $\begin{array}{c}\text { AHRS, } \\
\text { Kattalagere }\end{array}$ & $\begin{array}{c}\text { UAHS, } \\
\text { Shivamogga }\end{array}$ & $\begin{array}{c}\text { AHRS, } \\
\text { Honnavile }\end{array}$ & $\begin{array}{l}\text { ZAHRS, } \\
\text { Mudigere }\end{array}$ & $\begin{array}{c}\text { AHRS, } \\
\text { Bhavikere }\end{array}$ & $\begin{array}{c}\text { AHRS, } \\
\text { Ponnampet }\end{array}$ \\
\hline 2 & Latitude & $16^{\circ} 12^{\prime} \mathrm{N}$ & $13.054^{\circ} \mathrm{N}$ & $13.9299^{\circ} \mathrm{N}$, & $13^{\circ} 8^{\prime} 3^{\prime \prime} \mathrm{N}$ & $12.50^{\circ} \mathrm{N}$ & $12.14907^{\circ} \mathrm{N}$ \\
\hline 3 & Longitude & $74^{\circ} 54^{\prime} \mathrm{E}$ & $\begin{array}{c}75.03930^{\circ} \\
\mathrm{E}\end{array}$ & $75.5681^{\circ} \mathrm{E}$ & $\begin{array}{c}75^{\circ} 38^{\prime} 30^{\prime \prime} \\
\mathrm{E}\end{array}$ & $77.35^{\circ} \mathrm{E}$ & $75.94052^{\circ} \mathrm{E}$ \\
\hline 4 & Elevation & 598 meters & 569 meters & 570 meters & $\begin{array}{c}915 \\
\text { meters }\end{array}$ & $566.7 \mathrm{met}$ & 851 meters \\
\hline 5 & $\begin{array}{c}\text { Average } \\
\text { temperature }\end{array}$ & $25.5^{\circ} \mathrm{C}$ & $24.8^{\circ} \mathrm{C}$ & $24.6^{\circ} \mathrm{C}$ & $23.2^{\circ} \mathrm{C}$ & $36{ }^{\circ} \mathrm{C}$ & $22.6^{\circ} \mathrm{C}$ \\
\hline 6 & Average rainfall & $567 \mathrm{~mm}$ & $909 \mathrm{~mm}$ & $863 \mathrm{~mm}$ & $610 \mathrm{~mm}$ & $\begin{array}{c}1104.2 \\
\mathrm{~mm}\end{array}$ & $2173 \mathrm{~mm}$ \\
\hline
\end{tabular}


Table.3 Pooled ANOVA values for thirteen quantitative traits over six environments

\begin{tabular}{|c|c|c|c|c|c|c|c|c|}
\hline Source of Variations & DF & $\mathbf{X 1}$ & $\mathbf{X} 2$ & $\mathbf{X 3}$ & $\mathrm{X} 4$ & $\mathbf{X 5}$ & X6 & $\mathbf{X 7}$ \\
\hline Rep within Evn. & 6 & 0.82 & 13.52 & 4.62 & 0.53 & $1 . .67$ & 0.51 & 57.53 \\
\hline Varieties & 22 & $168.92 * *$ & $868.42 * *$ & $512.17 * *$ & $2.48 * *$ & $4.67 *$ & $5.29 * *$ & $522.81 * *$ \\
\hline Env.+(Var.x Env.) & 115 & $51.62 * *$ & 8.71 & $74.93 * *$ & $1.66 * *$ & $4.42 *$ & 1.71 & 184.72 \\
\hline Environments & 5 & $868.06 * *$ & 15.24 & $1293.62 * *$ & $25.77 * *$ & $35.45^{* *}$ & $8.56^{* *}$ & $845.53 * *$ \\
\hline Var.x Env. & 110 & 14.51 & 8.41 & 19.53 & 0.57 & 3.01 & 1.40 & 154.68 \\
\hline Environments(Lin.) & 1 & $4340.31 * *$ & 76.20 & $6468.11 * *$ & $128.86^{* *}$ & $177.27 * *$ & $42.81 * *$ & $4227.68 * *$ \\
\hline Var.x Env.(Lin.) & 22 & 18.42 & 4012 & 3.40 & 0.72 & 3.46 & 1.24 & 152.29 \\
\hline Pooled Deviation & 92 & 12.94 & 9.07 & $22.54 * *$ & $0.50 * *$ & $2.77 * *$ & $1.38 * *$ & $148.53 * *$ \\
\hline Pooled error & 132 & 0.66 & 3.39 & 8.69 & 0.23 & 0.96 & 0.87 & 65.71 \\
\hline Total & 137 & 70.45 & 146.77 & 145.14 & 1.79 & 4.46 & 2.29 & 23.01 \\
\hline
\end{tabular}

\begin{tabular}{|c|c|c|c|c|c|c|c|}
\hline Source of Variations & DF & $\mathbf{X 8}$ & X9 & $\mathbf{X 1 0}$ & X11 & $\mathbf{X 1 2}$ & $\mathbf{X 1 3}$ \\
\hline Rep within Evn. & 6 & 60.46 & 1.15 & 0.33 & 57622.57 & 139889.49 & 1.05 \\
\hline Varieties & 22 & $563.92 * *$ & $11.93 *$ & $10.48 * *$ & $3332866.56 * *$ & $2807513.59 *$ & $42.77 * *$ \\
\hline Env.+(Var.x Env.) & 115 & 157.35 & 7.37 & 1.92 & $296132.08 *$ & 479592.10 & 4.89 \\
\hline Environments & 5 & $694.63 * *$ & $23.30 * *$ & $4.67 *$ & $1437879.31 * *$ & $1873335.65^{* *}$ & 3.32 \\
\hline Var.x Env. & 110 & 132.93 & 6.65 & 1.79 & 244234.48 & 416240.12 & 4.96 \\
\hline Environments(Lin.) & 1 & $3473.16 * *$ & $116.52 * *$ & $23.49 * *$ & $7189396.54 * *$ & $9366678.25^{* *}$ & 16.61 \\
\hline Var.x Env.(Lin.) & 22 & 106.99 & 7.82 & $2.55^{*}$ & $435198.69 * *$ & $631680.12 *$ & 4.66 \\
\hline Pooled Deviation & 92 & $133.35 * *$ & $6.08 * *$ & $1.53 * *$ & $18750.23^{*}$ & $346624.47 * *$ & $4.82 * *$ \\
\hline Pooled error & 132 & 66.17 & 0.50 & 0.26 & 124084.59 & 187533.05 & 2.39 \\
\hline Total & 137 & 222.64 & 8.11 & 3.29 & 783782.87 & 853418.90 & 10.97 \\
\hline
\end{tabular}

$* \& * *$ Significant at $5 \%$ and $1 \%$ respectively

Where,

$\mathrm{X} 1$ Days to $50 \%$ flowering

X5 Number of Tillers per plant

X9 Panicles fertility (\%)

X13 Harvest index (\%)

X2 Days to maturity

X3 Plant Height

X6 Number of Productive tillers

X10 Test weight

X7 No. Of spikelets tillers

X11 Grain Yield (kg/ha)

X4 Panicle Length

X8 No. Of grains per panicle

X12 Straw yield 
Table.4 Mean and stability parameters in 23 advanced genotypes of Rice

\begin{tabular}{|c|c|c|c|c|c|c|c|c|c|c|c|c|c|c|c|c|c|c|c|c|c|c|}
\hline \multirow[b]{2}{*}{$\begin{array}{l}\text { SI. } \\
\text { No. }\end{array}$} & \multirow[b]{2}{*}{$\begin{array}{c}\text { Advanced } \\
\text { breeding lines }\end{array}$} & \multicolumn{3}{|c|}{ Days to fifty per cent flowering } & \multicolumn{3}{|c|}{ Days to maturity } & \multicolumn{3}{|c|}{ Plant height (cm) } & \multicolumn{3}{|c|}{ Panicle length $(\mathrm{cm})$} & \multicolumn{3}{|c|}{ Number of tillers per plant } & \multicolumn{3}{|c|}{$\begin{array}{l}\text { Number of productive } \\
\text { tillers per plant }\end{array}$} & \multicolumn{3}{|c|}{ Number of spikelets per panicle } \\
\hline & & Mean & $\mathbf{S}^{2} \mathbf{d i}$ & $\mathbf{B i}$ & Mean & $S^{2} d i$ & bi & Mean & $\mathbf{S}^{2} \mathbf{d i}$ & bi & Mean & $\mathbf{S}^{2} \mathbf{d i}$ & bi & Mean & $\mathbf{S}^{2} \mathbf{d i}$ & bi & Mean & $\mathbf{S}^{2} \mathbf{d i}$ & bi & Mean & $\mathbf{S}^{2} \mathbf{d i}$ & bi \\
\hline 1 & JB-1-11-7 & 96.83 & $1.61^{*}$ & 1.14 & 125.58 & -1.74 & 0.31 & 67.08 & $19.20^{*}$ & 0.77 & 19.28 & 0.24 & $0.15^{*}$ & 19.21 & -0.62 & 0.91 & 19.21 & -0.62 & 0.91 & 161.92 & $864.08 * *$ & -0.90 \\
\hline 2 & JB-1-20-2 & 97.33 & $10.99 * *$ & 1.14 & 128.33 & 2.73 & 0.96 & 77.66 & $12.65^{*}$ & 1.00 & 19.58 & -0.08 & 0.83 & 18.67 & -0.61 & 1.20 & 18.67 & -0.61 & 1.20 & 169.58 & 17.25 & 0.80 \\
\hline 3 & JB-1-22-1 & 96.83 & $27.02 * *$ & 1.07 & 127.08 & 4.84 & 0.78 & 82.44 & 6.05 & 1.06 & 19.59 & -0.18 & 1.06 & 18.24 & -0.16 & 2.17 & 18.24 & -0.16 & 2.17 & 162.25 & -31.93 & 0.86 \\
\hline 4 & JB-1-22-2 & 93.92 & $41.45 * *$ & 1.00 & 125.92 & -1.53 & -0.87 & 82.67 & 2.45 & 0.90 & 19.38 & -0.20 & 0.79 & 18.08 & -0.21 & 1.79 & 18.08 & -0.21 & 1.79 & 163.17 & -38.36 & 0.55 \\
\hline 5 & JB-1-22-3 & 96.25 & $8.15^{* *}$ & 1.14 & 126.75 & $8.60^{*}$ & 3.19 & 83.91 & 0.91 & 1.06 & 20.32 & $0.65 * *$ & 1.03 & 18.78 & 1.22 & 0.99 & 18.78 & 1.22 & 0.99 & 174.67 & 45.20 & 1.15 \\
\hline 6 & JK-1-7-5 & 95.50 & 0.65 & $1.40^{*}$ & 126.92 & $14.01^{* * *}$ & 2.15 & 80.89 & -3.71 & 1.15 & 19.37 & 0.14 & 1.01 & 19.33 & -0.79 & $1.54 *$ & 19.33 & -0.79 & $1.54 *$ & 163.50 & -37.32 & 1.17 \\
\hline 7 & JK-1-11-8 & 94.75 & $13.74 * *$ & 1.37 & 125.92 & 0.80 & 1.75 & 81.68 & -4.17 & 1.11 & 19.00 & -0.02 & 0.87 & 18.73 & -0.17 & 1.55 & 18.73 & -0.17 & 1.55 & 171.00 & $94.29 *$ & -0.35 \\
\hline 8 & JK-1-12-1 & 95.00 & $7.75^{* *}$ & 1.30 & 126.67 & 3.18 & 1.48 & 81.29 & 3.51 & 1.04 & 19.72 & -0.10 & 0.98 & 18.98 & -0.51 & 0.93 & 18.98 & -0.51 & 0.93 & 165.50 & -20.01 & 1.77 \\
\hline 9 & JK-1-13-1 & 96.67 & $11.30^{* * *}$ & 1.28 & 127.67 & $7.61 *$ & 2.58 & 79.91 & 4.02 & 0.98 & 19.37 & 0.20 & 1.26 & 19.13 & $1.66^{*}$ & 1.79 & 19.13 & $1.66^{*}$ & 1.79 & 170.33 & $112.46^{*}$ & 1.05 \\
\hline 10 & JK2-2-1-8-1 & 97.58 & $16.72 * *$ & 0.87 & 124.25 & 5.13 & 1.69 & 81.11 & 1.25 & 0.99 & 20.03 & -0.09 & 1.03 & 18.72 & -0.35 & 1.88 & 18.72 & -0.35 & 1.88 & 153.08 & 75.66 & 2.07 \\
\hline 11 & JK2-1-12-1 & 98.58 & -0.47 & 0.94 & 126.33 & $14.17 * *$ & 3.10 & 78.59 & -3.55 & 1.17 & 19.62 & $0.68 * *$ & 0.63 & 20.00 & 0.04 & -0.70 & 20.00 & 0.04 & -0.70 & 154.67 & 58.46 & 1.19 \\
\hline 12 & JA-4-1 & 99.83 & $7.46 * *$ & 0.81 & 120.42 & 0.73 & 1.50 & 84.12 & 5.95 & 0.86 & 19.95 & 0.02 & 1.05 & 18.95 & -0.23 & 1.31 & 18.95 & -0.23 & 1.31 & 159.67 & 13.48 & 0.20 \\
\hline 13 & JA-4-2 & 93.25 & $22.77 * *$ & 1.33 & 118.42 & 2.52 & 0.49 & 81.18 & -5.31 & 0.90 & 20.12 & $0.66 * *$ & 1.46 & 19.88 & 0.09 & 0.70 & 19.88 & 0.09 & 0.70 & 162.92 & -6.79 & 1.73 \\
\hline 14 & JA-4-3 & 95.67 & $9.55^{* * *}$ & 1.00 & 118.75 & -0.08 & 1.08 & 80.87 & -2.02 & 0.94 & 19.70 & -0.17 & $1.36^{*}$ & 19.58 & $1.50^{*}$ & 0.55 & 19.58 & $1.50^{*}$ & 0.55 & 162.83 & 17.08 & 2.16 \\
\hline 15 & JA-6-2 & 92.58 & 4.91 ** & $0.47^{*}$ & 118.58 & -2.68 & $-0.89 *$ & 82.58 & 0.08 & 0.84 & 20.45 & $0.74 * *$ & 0.61 & 21.10 & $1.99 *$ & -1.03 & 21.10 & $1.99 *$ & -1.03 & 178.33 & 28.69 & $\begin{array}{l}0.89 \\
\end{array}$ \\
\hline 16 & JA-6-3 & 95.17 & $7.29 * *$ & 0.68 & 119.17 & -2.11 & 0.53 & 80.38 & -5.70 & 1.01 & 19.34 & $0.50 *$ & 0.99 & 19.40 & 0.61 & 0.64 & 19.40 & 0.61 & 0.64 & 163.50 & $271.68 * *$ & 0.67 \\
\hline 17 & JA-6-4 & 96.92 & $19.00^{* *}$ & $0.05^{*}$ & 120.17 & -2.56 & 0.72 & 82.15 & -0.27 & 1.01 & 20.59 & $0.62 * *$ & 1.62 & 18.84 & 0.04 & 2.05 & 18.84 & 0.04 & 2.05 & 165.08 & -13.86 & 1.48 \\
\hline 18 & JT-2-15-1 & 102.75 & $11.46^{* *}$ & 1.22 & 149.67 & $14.60^{* *}$ & 0.18 & 100.85 & $36.12 * *$ & 1.08 & 20.16 & -0.08 & 1.06 & 19.88 & $3.63 * *$ & 1.42 & 19.88 & $3.63 * *$ & 1.42 & 162.58 & -30.54 & 0.12 \\
\hline 19 & JT-2-16-1 & 100.92 & $9.70^{* * *}$ & 0.84 & 149.08 & $22.75^{* * *}$ & -0.64 & 107.89 & $71.88^{* * *}$ & 0.95 & 20.69 & $1.75 * *$ & 1.85 & 19.23 & 1.06 & 0.15 & 19.23 & 1.06 & 0.15 & 155.67 & -52.95 & $-0.26 * *$ \\
\hline 20 & JT-2-22-5 & 98.83 & $6.71 * *$ & 0.87 & 149.67 & $18.33^{* *}$ & 0.23 & 102.12 & $102.80^{* *}$ & 1.07 & 19.77 & -0.05 & 0.96 & 20.31 & $3.21 * *$ & 0.26 & 20.31 & $3.21 * *$ & 0.26 & 166.58 & $302.55^{* *}$ & 1.26 \\
\hline 21 & Jyothi & 96.83 & $8.63^{* * *}$ & 1.21 & 132.17 & -3.16 & 0.19 & 78.16 & $19.08^{*}$ & 0.94 & 17.62 & 0.33 & 0.97 & 16.50 & 0.86 & 0.78 & 16.50 & 0.86 & 0.78 & 135.67 & $103.70^{*}$ & 2.98 \\
\hline 22 & KHP-2 & 109.92 & $9.67 * *$ & 0.81 & 153.25 & 3.17 & 1.33 & 82.84 & 35.39 ** & 1.22 & 19.14 & 0.26 & 0.52 & 17.58 & 0.56 & 0.53 & 17.58 & 0.56 & 0.53 & 148.00 & 39.32 & 2.05 \\
\hline 23 & Tunga & 116.00 & $26.21 * *$ & 1.06 & 155.67 & $11.29 * *$ & 1.14 & 98.78 & $26.14 * *$ & 0.97 & & & & 18.35 & -0.75 & 1.62 & 18.35 & -0.75 & 1.62 & 149.17 & $100.86^{*}$ & 0.38 \\
\hline & Mean & 98.17 & & & 130.28 & & & 84.31 & & & 19.66 & & & 19.04 & & & 19.04 & & & 161.72 & & \\
\hline
\end{tabular}

\section{Continued...}


Int.J.Curr.Microbiol.App.Sci (2018) 7(6): 1629-1638

\begin{tabular}{|c|c|c|c|c|c|c|c|c|c|c|c|c|c|c|c|c|c|c|c|}
\hline \multirow[b]{2}{*}{ SI. No. } & \multirow[b]{2}{*}{$\begin{array}{c}\text { Advanced } \\
\text { breeding lines }\end{array}$} & \multicolumn{3}{|c|}{ Number of grains per panicle } & \multicolumn{3}{|c|}{ Panicle fertility (per cent) } & \multicolumn{3}{|c|}{ Test weight $(\mathrm{g})$} & \multicolumn{3}{|c|}{ Grain yield (kg/ha) } & \multicolumn{3}{|c|}{ Straw yield (kg/ha) } & \multicolumn{3}{|c|}{ Harvest index } \\
\hline & & Mean & $\mathbf{S}^{2} \mathbf{d i}$ & $\mathbf{B i}$ & Mean & $\mathbf{S}^{2} \mathbf{d i}$ & bi & Mean & $S^{2} d i$ & bi & Mean & $\mathbf{S}^{2} \mathbf{d i}$ & bi & Mean & $\mathbf{S}^{2} \mathbf{d i}$ & bi & Mean & $\mathbf{S}^{2} \mathbf{d i}$ & bi \\
\hline 1 & JB-1-11-7 & 144.83 & $455.18^{* *}$ & -0.60 & 89.90 & $9.23 * *$ & 2.87 & 30.74 & 0.29 & -0.05 & 6026.63 & -92232.61 & 0.83 & 7942.50 & 128655.11 & 2.46 & 43.24 & 0.82 & 2.50 \\
\hline 2 & JB-1-20-2 & 153.95 & 46.69 & 0.38 & 90.78 & $8.69 * *$ & 1.42 & 30.06 & $5.85^{* *}$ & -0.28 & 6466.69 & $277102.89^{*}$ & 0.44 & 8093.33 & 117713.20 & 1.96 & 44.44 & $10.26^{* *}$ & 3.89 \\
\hline 3 & JB-1-22-1 & 145.16 & -42.84 & 0.65 & 89.51 & 12.11 ** & 0.29 & 31.52 & $0.62^{*}$ & -0.55 & 6490.38 & -102165.83 & $0.30^{*}$ & 8119.17 & 40851.44 & 1.31 & 44.48 & 1.34 & 0.14 \\
\hline 4 & JB-1-22-2 & 147.37 & 1.08 & 0.10 & 90.27 & $6.78^{* *}$ & -0.29 & 29.61 & 0.80 ** & -0.15 & 6530.04 & $175040.21 *$ & 0.22 & 8337.50 & 39571.17 & 1.97 & 43.95 & $8.49 * *$ & 4.90 \\
\hline 5 & JB-1-22-3 & 156.19 & $124.66^{*}$ & 1.36 & 89.28 & $10.81 * *$ & 0.50 & 29.81 & -0.08 & $2.64 *$ & 6459.36 & $303364.99 * *$ & -0.43 & 8346.67 & 35105.38 & -0.06 & 43.60 & 2.95 & 5.66 \\
\hline 6 & JK-1-7-5 & 147.33 & -31.59 & 0.87 & 90.13 & $6.05 * *$ & 2.15 & 29.62 & 0.20 & 2.66 & 6875.67 & $176756.09 *$ & -0.43 & 8728.33 & -41520.19 & 0.57 & 44.04 & 1.63 & 1.98 \\
\hline 7 & JK-1-11-8 & 152.93 & $240.29 * *$ & 0.09 & 89.24 & $9.04 * *$ & 1.56 & 29.75 & -0.11 & 1.68 & 6397.34 & -12557.51 & 1.24 & 8105.83 & $361806.04 *$ & 2.23 & 44.24 & 1.83 & 2.38 \\
\hline 8 & JK-1-12-1 & 144.50 & -54.80 & 1.35 & 87.41 & $6.65^{* *}$ & -0.99 & 30.64 & 0.04 & 1.35 & 6575.67 & 95776.87 & 1.26 & 8006.67 & 3470.32 & 0.70 & 45.11 & 1.76 & -0.25 \\
\hline 9 & JK-1-13-1 & 155.08 & 80.27 & 0.96 & 91.02 & 0.07 & $-0.48 *$ & 29.69 & 0.16 & 2.68 & 6549.13 & $244994.25 *$ & 1.54 & 8340.83 & -4518.89 & 0.70 & 43.90 & 0.95 & -0.81 \\
\hline 10 & JK2-2-1-8-1 & 138.13 & 82.61 & 1.96 & 90.21 & $5.04 * *$ & 2.56 & 30.11 & $1.62 * *$ & 2.80 & 6352.49 & -26221.86 & 1.29 & 8435.00 & 135465.77 & -0.78 & 42.90 & $3.88^{*}$ & 2.21 \\
\hline 11 & JK2-1-12-1 & 140.82 & 10.61 & 1.02 & 91.10 & $3.63 * *$ & 1.93 & 29.88 & 0.26 & 0.24 & 6177.71 & 54428.55 & 0.61 & 7824.17 & -11295.77 & 0.23 & 44.09 & -0.49 & 1.27 \\
\hline 12 & JA-4-1 & 145.70 & -6.66 & -0.02 & 91.23 & $1.23 *$ & 0.87 & 28.42 & 0.05 & $-1.32 *$ & 6634.93 & -11067.75 & 0.46 & 8472.50 & -34684.04 & -0.12 & 43.91 & 1.10 & 0.85 \\
\hline 13 & JA-4-2 & 146.95 & -32.34 & 2.22 & 90.11 & $1.77^{* *}$ & 1.46 & 30.27 & $2.23 * *$ & 1.42 & 6584.93 & -45167.91 & 0.63 & 8474.17 & -106266.60 & $-0.35^{*}$ & 43.73 & 0.51 & 2.29 \\
\hline 14 & JA-4-3 & 148.17 & 19.85 & 2.21 & 90.94 & 0.46 & 1.76 & 30.93 & $1.24 * *$ & 0.79 & 6696.80 & 66107.04 & 0.21 & 8395.83 & -152853.91 & 0.34 & 44.36 & -0.30 & 1.81 \\
\hline 15 & JA-6-2 & 167.83 & -4.39 & 1.12 & 94.12 & -0.18 & $-0.09 *$ & 30.76 & 0.05 & 0.08 & 6865.83 & -344.27 & 0.94 & 8599.17 & -143760.05 & 0.30 & 44.38 & -0.87 & 2.26 \\
\hline 16 & JA-6-3 & 146.17 & $163.47^{* *}$ & 0.95 & 89.50 & $6.30^{* * *}$ & 2.62 & 29.89 & $1.68 * *$ & 2.39 & 6477.90 & -69897.93 & 1.36 & 8460.00 & $276175.59 *$ & 0.90 & 43.44 & 1.17 & 0.51 \\
\hline 17 & JA-6-4 & 149.10 & -0.05 & 2.10 & 90.19 & $3.84 * *$ & 1.56 & 30.40 & $2.07 * *$ & 2.27 & 6456.39 & -2367.73 & 2.27 & 8963.33 & 9808.13 & 1.67 & 41.83 & 2.12 & 1.06 \\
\hline 18 & JT-2-15-1 & 146.47 & 5.54 & 0.78 & 90.01 & $4.54^{* *}$ & 2.05 & 30.09 & 0.31 & 1.02 & 7059.60 & $242504.26 *$ & 3.78 & 9891.67 & $848675.82 * *$ & 3.98 & 41.51 & $3.65^{*}$ & 1.14 \\
\hline 19 & JT-2-16-1 & 142.33 & -63.61 & $-0.29 * *$ & 91.43 & $2.28 * *$ & -0.76 & 29.32 & 1.48 ** & -0.55 & 6827.72 & $248044.72 *$ & 3.41 & 9737.50 & $547211.51 * *$ & 2.87 & 41.19 & 1.24 & 0.13 \\
\hline 20 & JT-2-22-5 & 152.17 & 341.57 ** & 1.45 & 91.25 & $9.03 * *$ & 2.16 & 30.51 & $3.02 * *$ & -0.25 & 7177.53 & 110844.45 & 2.81 & 10135.83 & $651461.76^{* * *}$ & 2.56 & 41.46 & 0.43 & -1.15 \\
\hline 21 & Jyothi & 118.42 & $181.04 * *$ & 2.39 & 87.22 & $4.80^{* *}$ & 0.95 & 25.09 & -0.07 & $-1.97 * *$ & 4093.06 & 52475.53 & 0.91 & 6994.17 & 50195.35 & 0.08 & 36.90 & $11.32^{* * *}$ & 0.34 \\
\hline 22 & KHP-2 & 132.73 & -49.05 & 1.55 & 89.94 & $12.96 * *$ & -1.42 & 27.37 & $6.20^{* * *}$ & 1.56 & 4881.40 & -81675.58 & 0.53 & 8443.33 & $471340.13 * *$ & -0.06 & 36.72 & 2.48 & 4.27 \\
\hline 23 & Tunga & 132.10 & 83.43 & 0.41 & 88.53 & $2.60 * *$ & 0.33 & 29.93 & $1.22 * *$ & $4.56^{*}$ & 4788.67 & -68369.08 & $-1.15 * *$ & 8680.83 & $484138.77 * *$ & -0.44 & 35.57 & 0.83 & $-5.83 *$ \\
\hline & Mean & 145.84 & & & 90.14 & & & 29.76 & & & 6323.73 & & & 8501.23 & & & 42.69 & & \\
\hline
\end{tabular}


The three advanced breeding lines JB-1-11-7 and JA-4-2 had more mean value for number of productive tillers per plant than population mean, also had regression coefficient value is around unity and very less deviation from regression. So, it is indicated that the genotype had stable performance across the environments. These findings are agreement with those of Vishnuvardhan et al., (2015). JA-6-2 and JT-2-22-5, JA-6-2 and JT-2-22-5, JT-2-15-1, JB-1-22-3 and JK-1-7-5, JK2-112-1 and JK2-1-12-1 are identified as stable lines The advanced breeding line JA-6-2 had more mean value for test weight than population mean also had regression coefficient value is around unity and less deviation from regression. So, it is indicated that this advanced breeding line had stable performance across the environments and less sensitive to environment it can adapt to the diverse environments. JA-6-4, JA-6-4, JA-42, JA-4-2, JB-1-20-2 and JA-4-2 are identified as stable lines for specific locations. This is on par with results of Deshphande et al., (2003), Arumugam et al., (2007), Panwar et al., (2008), Ramya and Senthilkumar (2008) and Krishnappa et al., (2009). Among twenty advanced breeding lines including checks line JA-6-2 had more mean value than population mean also had regression coefficient value is around unity and less deviation from regression. Therefore, it is indicated that this advanced breeding line had stable performance across the environments and it can adapt to the diverse environments. Hence, it can be used as stable line adopted across the environments and could be released for large scale trials. JT-2-16-1, JT-2-22-5, JA-4-3, JT-2-15-1, JB-1-20-2 and JK-1-7-5 are identified as suitable lines for specific locations. These results are also reported by Mall et al., (2013). The advanced breeding line JK-1-7-5 had more mean value for straw yield per hectare than population mean also had regression coefficient value is around unity and less deviation from regression. So, it is indicated that the advanced breeding line had stable performance across the environments and less sensitive to environment it can adapt to the diverse environments. JT-2-22-5, JT-2-15-1, Tunga, JT-2-15-1, JT-2-15-1 and JT-2-22-5 are identified as stable lines for specific locations. These findings are in conformity with Patil et al., (2013). High mean value than the population mean, regression coefficient around unity and least deviation from regression were recorded for harvest index in the advanced breeding line JA-4-1 indicating that their stability over wide range of environment.JK2-2-1-8-1, JK-1-12-1, JA-6-3, JB-1-22-2, JA-4-1 and JB-1-20-2 are identified as stable lines for specific locations.(Gourishankar et al., (2008), Ramya and Senthilkumar (2008) and Krishnappa et al., (2009)).

Different measures of stability have been used by various workers earlier, Finlay and Wilkinson (1963) considered linear regression slopes as a measure of stability. Eberhart and Russel (1966) emphasized the need of considering both linear and nonlinear component of Genotype $\mathrm{x}$ Environment interaction in judging the stability of genotypes. Later Breese (1969); Samuel et al., (1970); Paroda and Hayes (1971) and Jatasra and Paroda (1978) emphasized that the linear regression could simply be regarded as a measure of response of a particular genotype whereas deviation around the regression line was the most suitable measure of stability. In the present study the stability was assessed by the parameters suggested by Eberhart and Russel (1966).

The term stable genotype has been used for the average performance in all environments. Hence, such a stable variety has a high mean, unit regression and a minimum deviation from regression. From the present study it is concluded that genotypes JA-6-2 was found 
to be a stable across the environments and this genotype can also be used as a donor parent for generating new breeding material for development of variety with good stability for irrigated conditions. However, this needs to be verified by testing the breeding lines over the season and over the locations for one more year under rain fed condition.

\section{References}

Amrithadevarathinam, A., 1987, Stability analysis of some released varieties and local cultivars in dry and semidry condition. Madras Agric. J., 24 (10-11): 434-439.

Anonymus, 2016, FAO data, http//faostat.fao.org/.

Arumugam, M., M. P. Rajenna and B. Vidyachandra., 2007, Stability of rice genotypes for yield and yield components over extended dates of sowing under Cauvery command area in Karnataka. Oryza., 44:104-107.

Basavaraj, D.M., 1994, Stability analysis of promising genotypes of low land rice of hill zone of Karnataka. M.Sc (Agri) Thesis submitted to Univ. Agric. Sci., Bangalore. 58-75.

Deshpande, V. N., Dalvi, V. V., Awadoot, G. S., and Desai, S. B. 2003, Stability analysis in hybrid rice. J. Maharashtra Agri Univ., 28 (1): 87-88.

Eberhart, S.A., and Russell, W.A. 1966, Stability parameters for comparing varieties. Crop Sci., 6 (1): 36-40.

Ganesh, S. K., and Soundarapandian, G., 1987, Association studies for stability parameters in short duration varieties of rice (Oryza sativa L.). Madras Agric.J., 74: 208-212.

Gourishankar, V., Ansari, N. A., and Ilyas A., 2008, Stability analysis using thermosensitive genic male sterility (TGMS) system in rice (Oryza sativa L.). Res. on Crops, 9 (1): 141-146.
Kang, M.S., \& Martin, F. A. 1987, A review of important aspect of genotypeenvironmental interactions and practical suggestions for sugarcane breeders. $J$. of American Soci.of Sugarcane Techn., 7: 36-38.

Karnataka. Crop Res., 38:141-143.

Koli, N. R., Bagri, R.K., Kumhar, B. L., Chandra, P., Mahawar, R. K. and Punia, S. S., 2015, Assessment of stability performance in scented rice genotypes under transplanted condition of southeastern plain zone of Rajasthan. Electronic J. Plant Breed, 6 (4): 992995.

Krishnaappa, M. R., Chandrappa, H. M. and Shadakshari. H. G., 2009, Stability analysis of medium duration hill zone rice genotypes of Karnataka. Crop Res., 38:141-143.

Mahapatra, K.C. and Sujathadas, 1999, Stability of yield in relation to component traits in rice. Oryza, 36: 301-305.

Mall, A. K., Swain, P., Singh, O. N. and Baig, M. J., 2013, Use of Genotype x Environment Interactions and Drought Susceptibility Index for Identification of Drought Tolerant Genotypes at Vegetative Stage in Upland Rice. Indian J. Dryland Agric. Res Development, 27: 73-78.

Maurya, D.M. and Singh, D.P., 1977, Adaptability in rice. Indian J. Genet., 37:403-410.

Panwar, L. L., Joshi, V. N. and Ali, M., 2008, Genotype x environment interaction in scented rice. Int J. Rice., 45 (2):103-109.

Patil, A. B., Desai, R. T., Patil, S. A., chougule, Girish. R. and Shinde, D. A., 2013, Stability Analysis for Grain Yield and Its Component Traits in Rice (Oryza sativa L.). Trends in Biosciences., 6 (3):281-287. 
Praveen, S., Anil, P., and Rajesh, K., 2013, Stability study in aromatic rice (Oryza sativa L.). Crop Res., 45 (1-3):59-65.

Ramya, K. and Senthilkumar, N., 2008, Genotype $\mathrm{x}$ environment interaction for yield and its component traits in rice (Oryza sativa L.). Crop Improvement., 35 (1): 11-15).

Sawant, D. S., Kunkerkar, R. L., Shetye, V. N. and Shirdhankar, M. M., 2005, Stability assessment in late duration rice hybrids. In National seminar on "Rice and Rice Based Systems for Sustainable Productivity, Extended summaries., ICAR Research Complex for Goa", 1819th October. 75- 76.

Shrestha SP, Asch F, Dusserre J, Ramanantsoanirine A, Brueck H, 2012, Climate effects on yield components as affected by genotypic responses to variable environmental conditions in upland rice systems at different altitudes. Field Crops Res, 134:216228.

Singh, S. P., and Shukla, S., 2001, Stability parameters for opium and seed yield in opium poppy (Papaver somniferum L.). Indian J. Agril. Sci., 71:313-315.
Song, X.J., Huang, W., Shim, Z. M. and Lin, H. 2007, A QTL for rice grain width and weight encodes a previously unknown RING- type E3 Ubiquitin ligase. Nat Genet. 3(9): 623-630.

Subramanya, H., 1996, Stability analysis in rice (Oryza sativa L.) hybrids. M.Sc (Agri) Thesis submitted to Univ. Agric. Sci., Bangalore. 48-103.

Umadevi, M., Veerabadhiran, P. and Manonmani, S., 2008, Stability Analysis for Grain Yield and its Component Traits in Rice (Oryza sativa L.). J. Rice Res., 3 (1): 10-12.

Vanave, P. B., Apte, U. B., Kadam, S. R. and Thaware, B. L., 2014, Stability analysis for straw and grain yield in rice (Oryza sativa L.). Electronic J. Plant Breed., 5: 442-444.

Vishnuvardhan, B. R., Payasi, K., Devendra and Anwar, Y., 2015, Stability analysis for yield and its components in promising rice hybrids. Int Quarterly $J$. Envir sci., 9 (1-2): 311-321.

Yan W., Hunt, L. A., Sheng, Q., \& Szlavnics, Z. (2000). Cultivar evaluation and mega environment investigation based on the GGE biplot. Crop Sci., 40(3):597-605.

\section{How to cite this article:}

Manjunatha B., C. Malleshappa and Niranjana Kumara B. 2018. Stability Analysis for Yield and Yield Attributing Traits in Rice (Oryza sativa L.). Int.J.Curr.Microbiol.App.Sci. 7(06): 1629-1638. doi: https://doi.org/10.20546/ijcmas.2018.706.194 